\title{
THE ORIGIN OF THE RENAL ARTERY IN MAMMALS AND ITS ANOMALIES
}

\author{
JOHN LEWIS BREMER \\ From the Department of Anatomy, Harvard Medical School
}

TEN FIGURES

An embryological explanation of any anomaly should show that from some pre-existing embryological condition both the normal and the abnormal results may be derived; the agents which may cause the anomaly should be simple in themselves, as pressure, or the blocking of a vessel, or the relative overgrowth or arrest of development of certain parts, though the ultimate cause of these agents will usually remain a mystery. Not infrequently other vertebrates may develop normally in ways which for man would be abnormal, and the citation of such instances strengthens the explanation of any human anomaly.

Certain of the anomalies of the renal arteries have been already satisfactorily explained. Organs which make extensive migrations during growth from one position to another may either retain vessels from their original location, as in the case of the testis, or receive and incorporate new vessels of the region invaded, as does the thyroid gland. The instances of accessory renal arteries arising from the iliac arteries, from the middle sacral and inferior mesenteric arteries are to be considered as a persistance of the original renal vessels, normally lost, derived from the plexus between the vessels mentioned, lying directly in the path of the renal outgrowth from the Wolffian duct. This plexus has been injected in the pig embryo of $14.0 \mathrm{~mm}$. by Jeidell (1), and its influence commented on by her and by Evans (2); it is easily traceable in embryos of other mammals of similar age. Since it extends from side to side, and since the inferior mesenteric artery at this time arises by several roots from the aorta, 
one can readily understand the rare cases in which the right and left accessory renal arteries both spring from the inferior mesenteric artery, or both arise from a common stem from the aorta below that vessel. Such a case has been reported and figured recently by Harvey (3).

This class of anomaly may be considered thus separately because it has no relation to the question of the origin of the normal adult renal artery. The plexus described does, however, give a valuable clue to other anomalous conditions. What then is the origin of the permanent renal artery?

Broman (4) and others have stated that in man the renal artery is the trunk of a former mesonephric vessel, utilized secondarily as the inferior suprarenal artery, and finally as the renal artery, with the suprarenal as a branch. On the other hand, Hochstetter (5), Hill (6), and others have maintained that in mammals other than man, the renal artery is normally a new branch from the aorta at about the time when the kidney has reached its permanent position. Hill places this at $28.0 \mathrm{~mm}$. in the pig, at which age he succeeded in injecting this vessel, but states in another paper that in man the vascularization occurs between 22.0 and $24.0 \mathrm{~mm}$. No explanation has been offered for the peculiarity of man in this respect, and in fact very little corroborative work has been done on this subject. This is all the more strange because the conception that a new vessel can sprout from a well established trunk, as stated by Hochstetter and Hill, is contrary to the modern ideas of vessel growth. Yet other writers also, notably Kolster (7), at a loss to explain certain renal anomalies, have fallen back on the supposition of a 'late branch' from some neighboring vessel.

The aorta of pig embryos much smaller than $28.0 \mathrm{~mm}$. is provided with a well differentiated coat of modified mesenchyma, the future tunica media, through which the endothelium of a new branch would have to force its way. Now endothelium may and does sprout abundantly, but only in the looser, undifferentiated mesenchyma, or in its derivative, loose connective tissue. A slight condensation of the mesenchyma, as in the incipient somites, makes the.ingrowth of endothelial sprouts impossible, and 
causes the segmental arrangement of certain vessels. Equally, the condensation of mesenchyma around a vessel to form its coat should prevent any further outgrowth of endothelium to form new branches. Such a mesodermal wall, in a layer three or four cells thick, is found in human embryos at $10.0 \mathrm{~mm}$. and in the other mammals examined at about the same stage. The renal arteries, then, should be present before this stage, or, if later, must arise from the smaller and as yet uncoated aortic branches.

Following further this idea of the inability of endothelial sprouts to pierce condensed mesenchyma, we must recognize that the presence of a very slightly differentiated capsule around an organ will protect it from future ingrowth of blood vessels. Many vessels may become incorporated in the further development of the capsule as vessels to the capsule, not supplying the organ itself, but the fact that a vessel enters the organ proper, whether at the hilus or at some point on the periphery, indicates that the vessel was present and actively in use before the capsule was differentiated, and that the differentiation took place around the vessel. Wilson (8), in his study of hypernephromata, states that the renal capsule is distinct in man at $13.0 \mathrm{~mm}$., though not completely formed until much later, and my own observations agree with his on this subject. From the point of view of the ability of the kidney to receive, as well as of the aorta to send out new branches, we must, then, look for the renal vessels earlier than has been usually supposed.

We should not be deceived by the generally accepted idea of the great migration of the kidney and its relatively late arrival at its adult position. If we use the dorsal segmental arteries as a guide and the bifurcation of the aorta as a fixed point, and realize that the adult renal arteries arise opposite the third or fourth pair of dorsal arteries above this point (that is, the second or first pair of lumbar arteries in man), we find that the kidney has completed its migration with respect to its blood supply very early, at about $15.0 \mathrm{~mm}$. in man and pig, and at corresponding ages in the other mammals examined. Moreover, the permanent renal arteries are found often to enter at first the anterior pole of the organ, and secondarily to be moved by its actual 
enlargement to a position on its mesial border. The growth of the kidney itself and the rearrangement and growth of the surrounding parts complete the so-called migration and bring about the adult relations.

The problem thus resolves itself into the question, "What thin-walled arterial vessels are present in the embryo of roughly 10.0 to $15.0 \mathrm{~mm}$. in the area invaded by the kidney?"

Though the mesodermal wall of the aorta or of other vessels forms a barrier to new endothelial sprouts, it will naturally develop around any branches, however small, which are still in use. These smaller branches seem to have been overlooked heretofore, but, as I hope to show, are numerous and important in the present study. The aorta is usually considered to have certain sets of branches, called ventral, dorsal, and lateral or ventrolateral; but some confusion exists in the literature as to the distribution of these groups. The ventral vessels, originally bilateral, are derived from the vitelline plexus, and go to the intestinal tract; the paired dorsal branches, which may arise by a single trunk, supply the spinal cord, and usually also the body-wall; the lateral branches, originally body-wall vessels, are said to join the dorsal segmental arteries, and to be replaced by new lateral branches to the mesonephros, irregularly arranged in several vertical rows and not segmental. Yet later, other lateral branches arise in certain regions destined for the body-wall, represented by the subclavian artery. Dorso-lateral vessels may, in the thoracic region, supply the mesonephros, while in the abdominal region of the same embryo similarly placed arteries go to the body-wall, and the mesonephric vessels become ventro-lateral. Moreover, the arrangement varies at different ages and in different classes of mammals at any one age.

Such a lack of regularity implies, first, the absence of any specificity in the vessels and, second, some means whereby the vessels may change their position on the aortic circumference, or join with neighboring aortic branches. That vessels are not specific, do not go to the Wolffian body because they are ventro-lateral or dorso-lateral, but because they are best placed mechanically to supply the mesonephros, is shown strikingly in the reconstruc- 
tion of a part of the aorta in a sheep embryo of $5.6 \mathrm{~mm}$., in which branches from the left aorta have become right mesonephric arteries (fig. 1). The two aortae, right and left, are still partially separated by one of the bars or septa of mesoderm covered by endothelium frequently found where two vessels of irregular calibre are in the process of fusing. The left aorta at this point is much the larger, so that the bar is far to the right of the median line, as indicated by the position of the notochord and the root of the mesentery. Intestinal branches are lacking at this level, and we are left in doubt as to their relations; but two vessels, one large with many branches, the other much smaller, spring distinctly from the left aorta and pass to the right mesonephric glomeruli. If we consider the double aorta as a whole, these vessels are ventro-lateral, and thus normal in their distribution; but for the left aorta itself, since these branches are the nearest to the aorta of the opposite side, they are actually in the position of median ventral branches, ordinarily destined for the intestine. How frequently such irregularly placed vessels occur it is impossible to say, as all trace of their irregularity is lost with the absorption of the bars. It is evident, however, that aortic branches from the medial ventral angle of each aorta are not necessarily predestined to supply the intestine, but are governed by mechanical convenience; and also that, at this early stage, new sprouts may grow from the aortic endothelium at various points.

These new sprouts, not heretofore def nitely recognized except as new mesonephric arteries or subclavian arteries, are much more numerous than has been supposed and afford to the already present vessels the means of changing their position on the aortic wall and of forming new connections with other vessels. They are sent out by the aortic endothelium between the existing vessels, as the circumference and length of the aorta increase, and like all endothelial sprouts tend to branch and anastomose, both vertically and horizontally, with each other and with the existing aortic branches. The intervals between the aortic roots, old and new, of the resulting plexus, and the size of its mesh are roughly the same as those f gured by Clark (9) in his studies 
on growing lymphatic vessels, and as those found by injections of blood vessels by Evans (2) and others. It is my idea that endothelium normally sends out sprouts at right angles to the main stem at fairly regular intervals, in the same way that the main stem of some trees sends out branches, and that in consequence the endothelial mesh throughout its growing portion is originally of a definite size. As this size is increased by the general growth of the body, carrying the capillaries further and further apart, new branches are given off between the old ones in order to regain the normal mesh size. This applies equally to the aorta, the circumference of which is, with growth, successively large enough to accomodate more and more branches with the normal interval between them, until, at the age when the mesodermal coat develops and stops further outgrowths, there are perhaps twelve or fourteen such branches, equally spaced, around the circumference. In the vertical plane there may be two or three sets of branches to each segment before the advent of the mesodermal coat. The later growth of the aorta, which may be greater in one part than in another, would separate these branches further from each other, but no new intervening vessels could be formed.

An anastomosing periaortic net having many connections with the aorta and linking the earlier aortic branches would result. Portions of such a net can be found occasionally, as certain of the accompanying figures show; part has already been injected by Jeidell, as already mentioned. Normally, the greater part of it degenerates very early. That it has not been more fully injected I attribute to the fact, as already suggested by me (10), that new endothelial vessels may be solid cords, even while connecting with hollow vessels or vesicles. Tracing such cords in serial sections and amongst mesenchymal cells is extremely difficult, though portions of the plexus, but not its connections, are recognizable in almost all the young embryos I have examined. Even the point of origin of the smaller roots from the aorta is hard to find, and I imagine that in many cases they may be absent in the prepared specimen because they have retracted from faulty preservation or under the influence of the 
fixing fluid, leaving only slight irregularities in the endothelial wall. In many stained and sectioned embryos the endothelium of the aorta is in festoons with few points of contact with the mesodermal layers; such points of contact correspond to the positions of the roots of this plexus, as found in better preserved specimens.

The aortic wall of condensed mesenchyma will, as has been said, naturally form around such of the vessels of this plexus as are still in use, and thus frequently portions of the horizontal and vertical vessels are buried deep in the wall. The parts of the net not in use will retract or otherwise disintegrate, leaving perhaps no trace of their former position and connections. Figure 2 shows several of these smaller aortic branches, two between the dorsal segmental artery and the mesonephric artery, others ventral to the latter. A portion of the net remains as anastomoses between some of the more ventral, and together with the roots is partially buried in the mesodermal coat of the aorta. The more dorsal of the roots have been carried further apart by the growth of the aortic wall, have lost their connections with the net, and one of them has become practically obliterated, leaving only a point of attachment of the endothelium to mark its position. The tips of two of the roots lie in the loose connective tissue and can send out further sprouts. Figures 3 and 4 show a portion of the periaortic net in an older embryo; the persisting vessels in this case are the anastomoses near the aorta, and the capillaries from them peripherally. Though the intermediate roots from the aorta are absent, the position of these peripheral capillaries indicates, to my mind, that the roots were originally three in number between the dorsal segmental artery and the mesonephric artery. Figure 5 shows the wealth of partly buried vessels found in the dorsal region of the aorta, representing anastomoses, both vertical and horizontal, between dorsal segmental and lateral aortic branches, and indicating that two sets of roots per segment in the vertical direction is the general arrangement in this case.

These are only a few of the many instances in which $I$ have found remains of this periaortic plexus. Similar connections between mesonephric arteries and ventral arteries are very com- 
mon (fgs. 8 and 10). Also anastomoses between paired dorsal segmental arteries are frequently found, and account for their later single trunk without the supposition that the two vessels fuse. The paired aortae, in the cervical region, may even send vessels toward the median line to form a plexus, especially well developed in the cat.

Through such a plexus any one of several paths may be chosen for a permanent vessel, and until the actual degeneration of the roots has taken place such a path may be moved from one position on the aortic wall to another. The mesonephric arteries of adult selachians are said by Dohrn (11) to arise normally from the lumbar arteries; a glance at f gure 4 will show how this could come about. Even the extreme case of Bdellostoma, in which the adult mesenteric arteries arise from the dorsal wall of the aorta in common with the dorsal segmental arteries, is explicable.

With the general growth of the area around the aorta, or with the special lengthening of any of its main branches, the anastomotic vessels of the plexus may come to lie outside the aortic wall, and the regular meshes of the net become thus distorted.

Fig. 1 Sheep embryo, $5.6 \mathrm{~mm}$. H.E.C. No. 1332, Sect. 220-235. Reconstruction of part of the abdominal aorta to show right mesonephric arteries from left aorta. nch., notochord; mes., root of mesentery; m.art., mesonephric arteries. $\times 100$ diam.

Figs. 3 and 4. Pig embryo, 24.0 mm., H.E.C. No. 1846, Sect. 1585-1610. Lateral view and semi-diagrammatic transverse section of a reconstruction of a portion of the abdominal aorta and periaortic net. The common trunk of the dorsal segmental arteries, d.arl, is connected within the aortic wall with a mesonephric artery, m.art. An intervening branch, $x$, from the aortic eavity has lost its connection. The lateral branches of the plexus suggest at least one more intervening branch to complete the net (cf. fig. 2), $\times 40$ diam.

Fig. 5 Pig embryo, 24.0 mm., H.E.C. No. 62, Sect. 1375-1525. Reconstruction of the dorsal aspect of abdominal aorta, to show anastomoses within and without the aortic wall between the dorsal segmental arteries and lateral aortic branches, one of which, $k$, arises as an intercalated dorsal vessel, runs to a lateral position, and emerges laterally to reach the kidney. There are two sets of dorsal branches per segment. d.art., dorsal segmental vessels, some with common trunk, some paired though connected. $\times 40$ diam.

Fig. 10 Man, $16.4 \mathrm{~mm}$., H.E.C. No. 1707, Sect. 774 858. Explanation in text. coe.a., coeliac axis; s.m.art., superior mesenterie artery; supr.art., suprarenal artery; r.art, renal artery. $\times 60$ diam. 

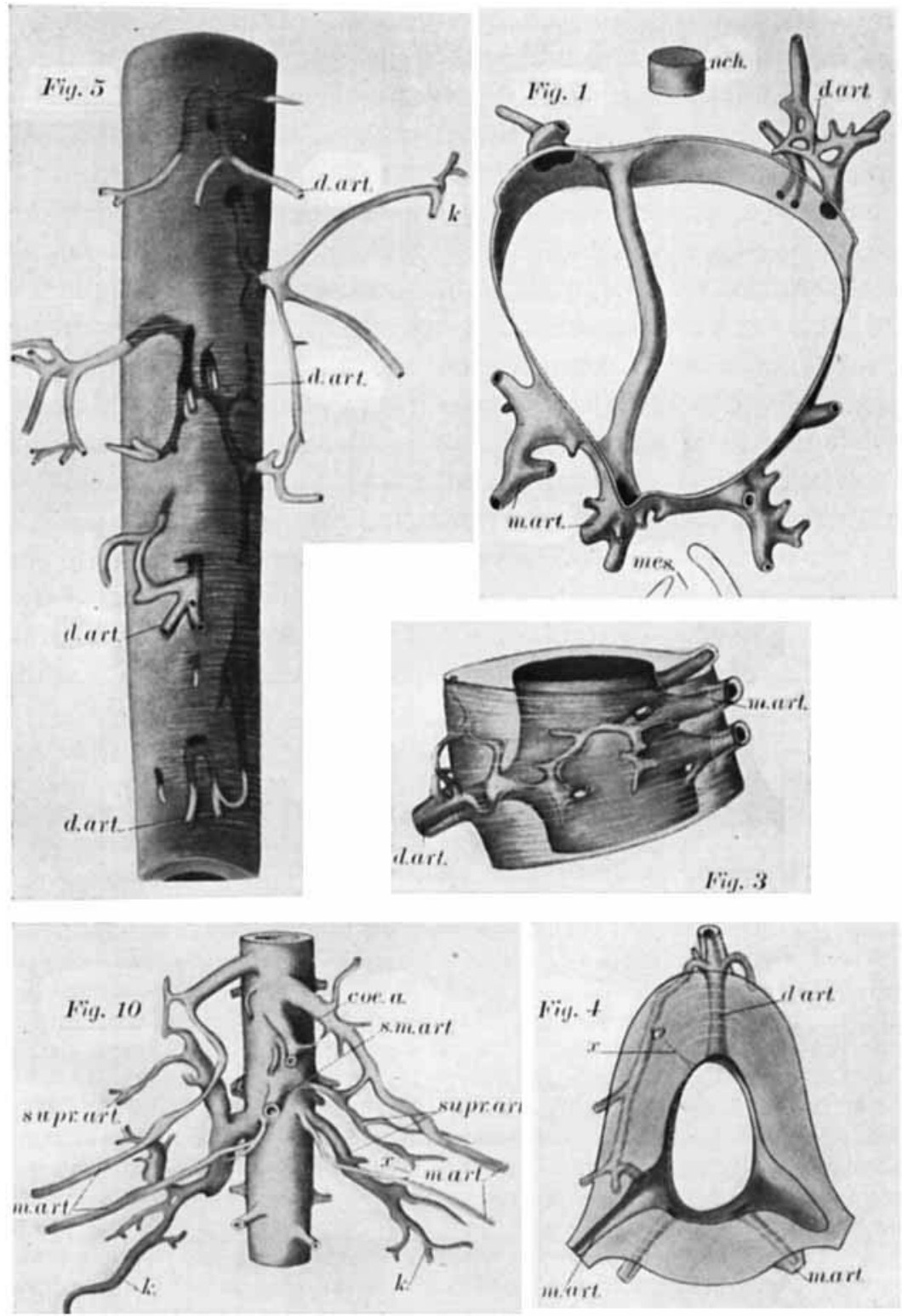
If this takes place after the main aortic branches are coated, no new sprouts will arise from them to form new anastomoses, and an arca near the aorta will be left which receives no blood supply from the larger aortic branches. Into this area grow sprouts from some of the discarded roots of the original plexus, already protruding beyond the aortic wall, but not strictly to be considered new aortic branches, because they were present before the aortic coat developed. Such rejuvenated vessels become the small arteries to the lymph glands and sympathetic ganglia near the aorta, the bronchial and oesophageal arteries, and by recurrent branches the vasa vasorum of the aorta itself. They, or the plexus of which they were a part, also send twigs to the organs developing in or migrating into the area immediately lateral to the aorta, namely the suprarenal gland and the kidney.

Which part of the plexus between the mesonephric arteries and the dorsal segmental arteries will remain, and what its connections will be, depends apparently on mechanical forces brought into play by idiosyncrasies of development in the different classes of mammals. Of the embryos examined in this study,

Fig. 2 Riabbit embryo, $10.0 \mathrm{~mm}$., 14 days, H.E.C. No. 155, Sect. 457. To show small aortic branches between the larger ones, and part of the periaortic plexus. The mesodermal aortic wall is present, and has partly buried the smaller branches. At ' $\mathrm{x}$ ' an obliterated branch. d.art., dorsal segmental artery; m.art., mesonephric artery. $\times 165$ diam.

Fig. 8 Cat embryo, $10.7 \mathrm{~mm}$., H.E.C. No. 474, Sect. 434-452. Reconstruction of the aorta at second lumbar segment; lateral plexus, $p l$., with mesodermal coat connected, at an acute angle, with a mesonephric artery, m.art., by a thick walled vessel, con., and with the aorta by three slender vessels of endothelium only, arranged in two vertical rows. Branches to body-wall and to kidney, $k$. Note portions of ventral plexus toward superior mesenteric artery, s.m.art. The left side of the drawing is incomplete (cf. figs. 6 and 9). $\times 130$ diam.

Fig. 9 Cat embryo, $11.6 \mathrm{~mm}$., H.E.C. No. 1979, Sect. 308. Drawing of the aorta and the left permanent renal artery, r.art., just enlarged and with simple endothelial wall, from the aorta to the plexus, $p l$. The plexus is coated; its connection, con., with the mesonephric artery, m.art., is obliterated and can be followed no further in neighboring sections. The renal branch of the plexus, $r . b r$. , enters the kidney, $k$, at a lower level. On the right side is seen a lateral branch, lat.br., not now continued to the right plexus, and dorsal to it the remains of another. ve., veins; sym., sympathetic nerves; b.u., branch to body-wall. $\times 165$ diam, 

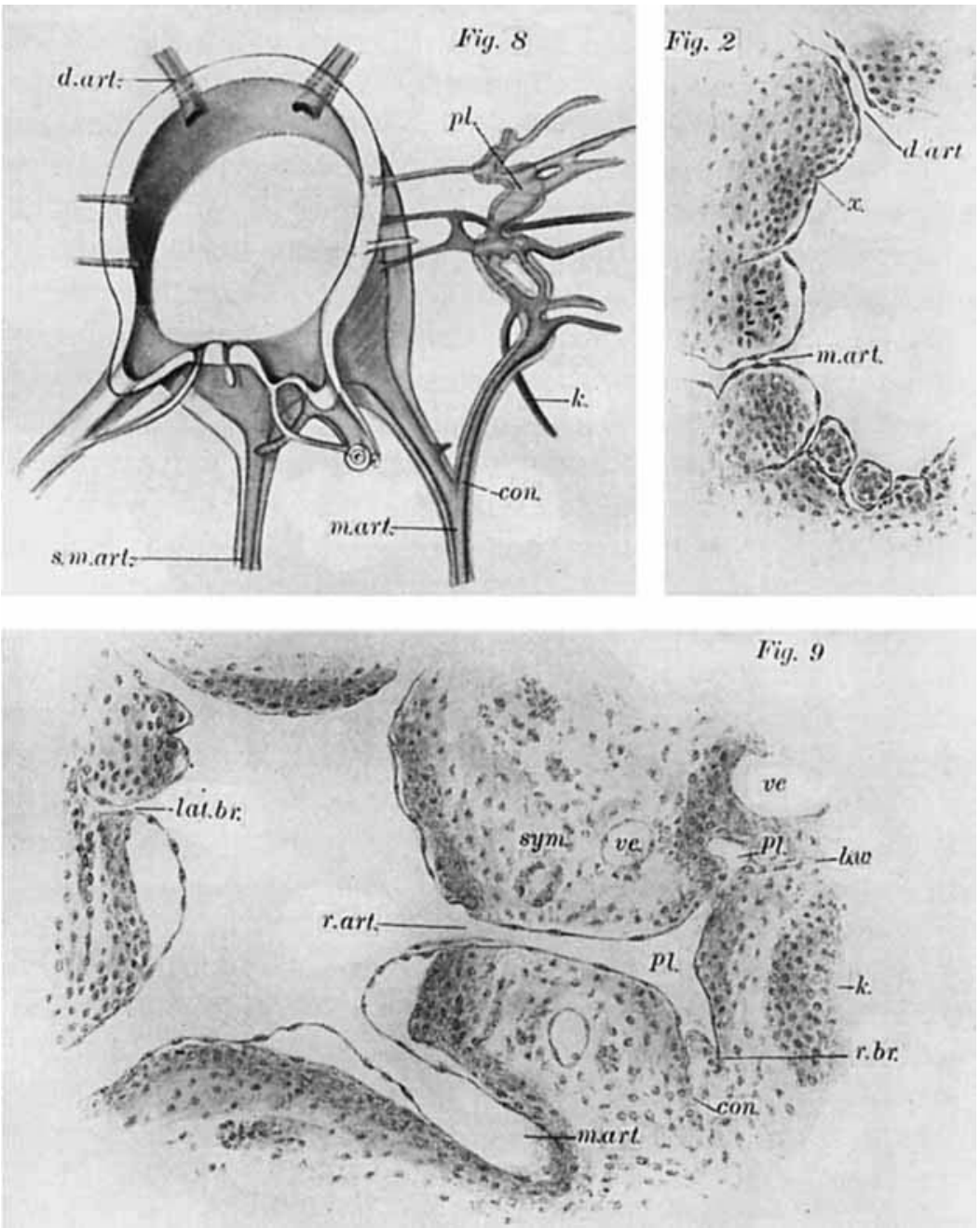
namely pig, rabbit, sheep, cat, and man, the two former have relatively a large Wolffian body, both in length and thickness, from a very early embryonic period. The increasing bulk of Wolffian body itself is enhanced by the consequent increase in the subcardinal veins, and these two factors cause a great protrusion of the organ into the coelom. The glomeruli, occupying the ventro-mesial border of the organ, are thus moved from their original position lateral to the aorta to one far ventral; and the mesonephric arteries, running to the glomeruli, are lengthened and arise from the ventro-lateral surface of the aorta, instead of from the lateral. This is true, to some extent, in all mammals, but occurs earlier and is exaggerated in the pig and the rabbit. The horizontal anastomoses of the plexus leading dorsally from the mesonephric arteries either do not form, or if already present are strained until they become obliterated, leaving the mesonephric arteries unconnected with the plexus, and far ventral to the area in which the kidney is developing (see diagram, fig. 7).

The fact that the spermatic artery is originally a branch from one of the arteries to the glomeruli of the Wolffian body, or as stated for the pig by Hill (6), a new vessel of the same type and with a similar course, gives us a check on the origin of the renal artery, for, if the renal artery is connected in any way with the mesonephric plexus, it will be common to find, as an anomaly, the spermatic artery as its branch. Such a connection I have not found described for either rabbit or pig.

A second factor which distinguishes different types of embryos is the greater or lesser curvature of the rump region. A comparison of the profile drawings of cat and rabbit embryos of about $10.0 \mathrm{~mm}$. or younger with those of man, sheep, or pig of the same age shows in the former a strikingly increased curvature of the back just anterior to the pelvic limb buds (fig. 6). A study of sections of these embryos shows that the aorta does not follow this curve closely, but takes a shorter path, and so lies here further from the spinal cord than in the thoracic region. This causes the dorsal segmental arteries of the lumbar aorta, which run to the spinal cord, to assume a course almost di- 


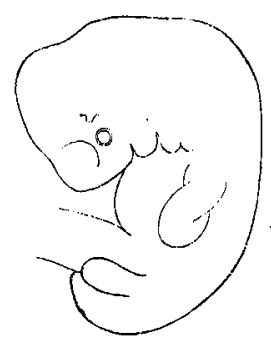

$A$

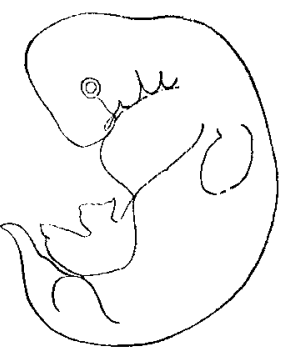

$B$

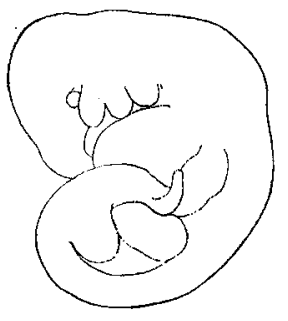

C

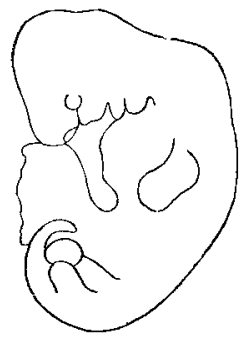

D

Fig. 6 Profile drawings of embryos to show the difference in the curvature of the rump region. A, man, $8.0 \mathrm{~mm}$., H.E.C. No. 817 ; B, pig, $9.0 \mathrm{~mm}$., H.E.C. No. 52; C, rabbit, $6.6 \mathrm{~mm}$., H.F.C. No. 460 ; D, eat, $9.7 \mathrm{~mm}$., H.E.C. No. 446.

rectly dorsal, instead of lateral or dorso-lateral, from the aorta. In this case the dorsal portion of the periaortic net will be distorted and obliterated, as was the ventral portion in animals with a large Wolffian body, and in the cat and the rabbit the dorsal segmental arteries may be expected to take no part in the development of the renal or suprarenal arteries (see diagram, fig. 7).

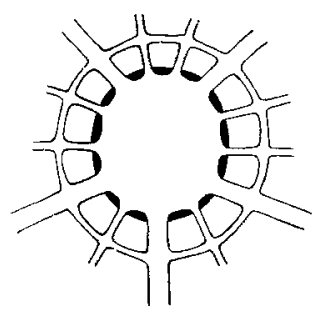

A
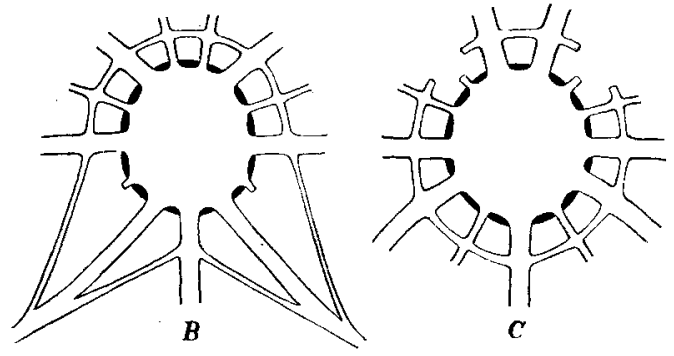

C

Fig. 7 Dingram to show, A, roots from the aorta between the dorsal segmental, mesonephric, and mesenteric arteries, and the periaortic plexus; $B$, result of large Wolffian body, carrying forward the mesonephric arteries; $\mathrm{C}$, result of greater curvature of back, lengthening the dorsal arteries. In both cases lateral branches enlarge to supply the fields left vacant.

It will be noted that the rabbit is mentioned in both of these classes, and should therefore be restricted to a lateral origin of these arteries, unconnected with either mesonephric or dorsal segmental vessels; anomalies should be very rare. Sheep and 
man, on the other hand, have neither large Wolffian body nor great curvature; less marked variations or the accidental blocking of one or the other of the vessels might be expected to determine the adult relations, and anomalies should be frequent and very variable.

A secondary result of the increased curvature of the rump region is the formation of the a. ilio-lumbalis and a. adrenolumbalis in the cat, and of similar vessels in the rabbit. These vessels, situated in the lumbar region, are derived from the extension of the periaortic plexus into the area lateral to the aorta, left vacant by the separation of the aorta and the spinal cord. In the thoracic region of these animals and in all regions of other animals this area is supplied by the ventral branches of the dorsal segmental arteries, which are prolonged into the lateral body-wall. But the increasingly dorsal direction of the dorsal arteries, following the increased curvature of the body, causes a sharp angle where the ventral branches leave their dorsal segmental trunks, and thus puts the ventral or body-wall branches at a disadvantage, and makes their territory of distribution fall an easy prey to the rapidly growing branches of the plexus with its shorter and more direct lateral roots from the aorta. The resulting vessels, as described in the cat by Reighard and Jennings (12), pass in front of the psoas muscles, and after anastomosing with each other (the longitudinal strands of the plexus) send branches to the ventral body muscles. In this region the usual ventral branches of the lumbar arteries are lacking.

Such vessels to the ventral and lateral body muscles should not be mistaken for the normal lumbar arteries of the adult, which are the dorsal segmental arteries of the embryo. The latter, or true lumbar arteries, pass behind the psoas muscles near to the vertebrae, to which they send branches, and then supply the spinal cord and dorsal muscles on their way to the body-wall. The newer vessels pass in front of the psoas group and are without dorsal branches. Because of the connections of both with the body-wall, I think their differences have often been overlooked in the descriptions of the anomalous origin in man of the renal artery from a 'lumbar artery.' 
The actual origin of the renal artery is found to correspond in the different groups of animals with the principles just stated. In cat embryos of 6.0 to $7.0 \mathrm{~mm}$. a well developed net exists, opposite the four lumbar segments, beside the aorta, connecting the mesonephric vessels, which have an almost lateral course, with small lateral sprouts from the aorta, and those again with the dorsal segmental vessels. With the increased curvature of the body these dorsal connections are lost, new sprouts from the lateral portion of the plexus extend toward the lateral wall, and a main channel, from a mesonephric artery of the first or second lumbar segment, is formed and becomes important enough to assume a mesodermal coat. The lateral connections of the plexus with the aorta remain small and uncoated. In a $10.7 \mathrm{~mm}$. cat embryo (H.E.C. No. 474) other branches from the lower end of this plexus have entered the superior pole of the kidney, which is still without a capsule (fig. 8). The renal blood supply may thus be said to be derived temporarily from the mesonephric artery, since the plexus receives most of its blood from this source. Meanwhile the growth of the Wolffian body, aided perhaps by the advent of the kidney dorsal to it, has forced the glomeruli to a more ventral position and lengthened the mesonephric arteries, among them the one which sends off the main coated afferent of the plexus. The result is that a sharp angle is formed between the mesonephric artery and its branch to the plexus, and that the smaller, lateral roots of the plexus become by far the most direct pathways to the plexus and hence to the kidney. One or more of these becomes enlarged, as is shown on the left side in an embryo of $11.6 \mathrm{~mm}$. (H.E.C. No. 1979). The lateral aortic branch is of large caliber (fig. 9), and connects the coated aorta with the coated plexus, yet is it. self provided with only an endothelial wall, showing its sudden rise to importance. The older main channel to the plexus from the mesonephric artery has become discontinuous in this case, and the other lateral branches from the aorta to the plexus, not seen in this section, have also lost their connections and remain as vessels to the immediate periaortic tissues. On the right side of this section one of these small vessels may be seen. 
The plexus here is still connected with the mesonephric arteries, and no enlargement of the lateral aortic branches is found, so that the right side of this embryo is in the same condition as both sides of the embryo of $10.7 \mathrm{~mm}$.

The permanent renal artery in the cat is thus formed partly from a suddenly enlarged but long present lateral branch of the aorta, partly from the longitudinal meshes of the periaortic plexus, and partly from branches from the lower end of this plexus to the upper pole of the kidney; the connections are present before $10.7 \mathrm{~mm}$.

Anomalies to be looked for in the cat, easily explicable by the history as thus shown, are, first, double or multiple renal arteries from the aorta, since there are several lateral connections from aorta to plexus, and, second, a spermatic or ovarian branch of the renal artery. This latter would require merely the persistence of the original mesonephric union with the plexus, and a reversal of the blood current through it, so that the mesonephric plexus from which the spermatic artery sprouts would receive its blood through the renal artery and the lateral plexus; the mesonephric trunks might then all disintegrate. Spermatic branches of the renal arteries are mentioned as occasionally present in the cat by Reighard and Jennings.

The renal artery in the rabbit has been figured by Lewis (13) in reconstructions of embryos of $11.0 \mathrm{~mm}$., 14 days (H.E.C. No. No. 1327), and $14.5 \mathrm{~mm}$., 14 days, 18 hours. (H.E.C. No. 143), in his paper on the development of the vena cava inferior. Though hardly referring to this artery in the text, he thus correctly represented it in embryos younger than those in which it had then been reported. It is shown as a longitudinal vessel, reaching the kidney at its caudal end, attached near the middle of its length, in the $11.0 \mathrm{~mm}$. embryo, by a vessel springing from the aorta midway between the dorsal segmental arteries and the mesonephric arteries. With more detailed study of rabbit embryos with this point of view, it is possible to find the periaortic plexus present very early, but it is partially lost in embryos of $8.0 \mathrm{~mm}$. to $9.0 \mathrm{~mm}$., leaving only the lateral sprouts from the aorta (cf. fig. 2). There are many of these in the lumbar region, 
connected, by longitudinal anastomoses, but unconnected with the mesonephric or dorsal segmental arteries for reasons stated above. From the longitudinal strands of the plexus branches pass to the body-wall, which by $10.0 \mathrm{~mm}$. (H.E.C. No. 155) have become long vessels. In this same embryo branches from the lower end of the plexus have entered the kidney. The longitudinal anastomosis and one of its aortic connections are the vessels figured by Lewis. The lateral branches to the bodywall are not shown by him, but nevertheless form a permanent connection; one at least remains as a normal branch of the renal a tery in adult rabbits. This is mentioned by Gerhardt (14) in the following description, p. 245: "From each renal artery, near its root springs a small (the 12th) intercostal artery." This is obviously not a true intercostal with dorsal connections, but must run in front of the psoas muscles and thus be comparable with the 'false lumbar' arteries already referred to. Spermatic branches of the renal artery are not described in the rabbit, to my knowledge, and are not to be expected because of the early separation of the plexus from the mesonephric arteries.

I think the detailed description of the origin of the renal artery in the cat and the rabbit is sufficient to show how the variations in the different classes arise. The pig makes use of a lateral aortic branch, or, as is shown in figure 5 , of a branch of the dorsal segmental artery. In the sheep a lateral aortic branch is commonly enlarged. In both the connection of the plexus with the kidney is made early, and originally at the superior pole of the organ.

It should be noted that in all classes of mammals the early position of the kidney, before it has escaped from the crotch of the lower bifurcation of the aorta by migrating upward and backward, as described by Pohlman (15) and by Lewis and Papez (16), is nearer to the mesonephric arteries, because more ventral, than after this escape. My observations, contrary to those of Broman (4) in his paper on the renal portal system, show frequent early mesonephric branches to the kidney, some in use, some partially obliterated, in all the animals examined. When no larger branches are present, certain of the glomerular efferent 
vessels of the Wolffian body can be traced toward the renal blastema. At no time does the kidney seem to be without arterial blood, from one source or another. The persistence of any of these mesonephric connections would make possible, as explained above, the presence of a spermatic branch of the renal artery; but an anomaly from this source must necessarily be extremely rare in animals with large Wolffian body, because it would depend upon the blocking of the more direct channels.

In man there is neither an excessive curvature of the body nor a large Wolffian body, so that theoretically the renal artery could be derived from the mesonephric, lateral, or dorsal segmental roots of the plexus; yet normally it is not directly derived from any of these. The reason for this is found in the precocious development of the suprarenal gland in man.

Goormaghtigh (17) finds the first growth of the mesothelial cords which form the cortex of the suprarenal gland at about ten days in the white mouse, about twenty days in the guineapig, but states that these cords do not separate from the mesothelium to form a recognizable organ until much later. Substituting the white rat for the white mouse, of which no specimens were available, I find that the gland is first seen as a large organ at about 14 days, $8.0 \mathrm{~mm}$.; in the guinea-pig it occurs in embryos of about the same length. In the cat the suprarenal cortex can be recognized as such at about $11.0 \mathrm{~mm}$., in the pig at 15.0 and at corresponding ages in the rabbit and sheep. In all of these animals, the organ occupies at this time the upper abdominal region, so that there is a long distance, representing five or six segments, between the upper pole of the kidney and the lower limit of the suprarenal gland.

The suprarenal arteries are also derived from the perioartic plexus or, if this has partially broken down, from its aortic roots. Thus in a cat embryo of $15.6 \mathrm{~mm}$. (H.E.C. No. 1983) an upper suprarenal artery springs from a loop between a dorsal segmental vessel and the coeliac axis. The mechanical forces which influence the choice between the aortic roots are similar to those explained for the renal artery, but may differ in the upper and lower levels of an organ which becomes as long as does the supra- 
renal gland. The rabbit utilizes as an inferior suprarenal artery one of the upper lateral branches of the plexus, from the lower end of which the renal sprouts develop, so that the suprarenal artery, as well as the 12 th intercostal artery, is normally a branch of the renal, as stated by Gerhardt. Other animals may or may not have such connections between renal and suprarenal arteries, but in all cases the renal is the older vessel.

In contrast to the other mammals examined the early development and rapid growth of the suprarenal gland in man is very striking. At $8.0 \mathrm{~mm}$. (H.E.C. No. 877) while the kidney is still a pelvic organ, the group of cortical cords has already grown down to the level of the first lumbar arteries, or in other words is occupying the region later to be invaded by the kidney. It would be interesting to speculate, in connection with the work of Cannon (18), on the activity of this gland in various emotions, whether man is given an advantage over other mammals by its precocious development; but that is at present beside the question. The result, so far as the arteries are concerned, is that the suprarenal gland primarily receives the vessels which in other mammals go to the kidney. These arteries are at first numerous, but only three survive, as was pointed out by Broman (4); and it is only the most caudal of the three which is immediately interesting. Broman states that the suprarenal arteries are branches of the mesonephric vessels, but I must disagree partially with this statement, for I have seldom seen such a connection in the embryos examined. Normally all of the suprarenal arteries are the lateral aortic roots of the plexus, similar to the renal arteries already described in many mammals. They never, in my specimens, extend to the mesonephric glomeruli, nor are they branches of vessels which do, though they are not infrequently connected by the anastomoses of the plexus with the mesonephric arteries. Exceptionally a mesonephric artery does send a small branch to the suprarenal gland but in the few cases I have seen, such branches are much smaller than the normal arteries, though probably capable of persisting under certain conditions. 
The mesonephric arteries themselves in man differ from those of other animals in the younger stages in that they are much distorted and bent from their usual direct course by the growth amongst them of the precocious suprarenal gland. Some are deflected ventrally, some dorsally, so that they arise in two more or less distinct vertical rows from the ventro-lateral and dorso-lateral surfaces of the aorta, respectively. The suprarenal vessels are the aortic roots between these two rows. The infrequent branches of mesonephric arteries to the suprarenal gland may approach it from behind or from in front.

An ascending branch from the upper suprarenal artery, or from the upper end of the plexus, frequently becomes the inferior phrenic artery; one or both of these vessels may be connected with the coeliac axis or with a lumbar artery (cf. cat $15.6 \mathrm{~mm}$., mentioned on p. 196). A descending branch from the lower suprarenal artery, or from the lower end of the plexus becomes the permanent renal artery. This vessel can be traced to the kidney in a human embryo of $16.0 \mathrm{~mm}$. (H.E.C. No. 1322), and in one of $16.4 \mathrm{~mm}$. (H.E.C. No. 1707). Terminal branches can be seen at the upper pole of the kidney in an embryo of 13.6 mm. (H.E.C. No. 839), but I am unable to trace with certainty the course of the vessel in this specimen.

In the $16.4 \mathrm{~mm}$. embryo (fig. 10), the more ventral mesonephric arteries sweep ventrally in front of the suprarenal gland, and then turn laterally to reach the glomeruli. Dorsal to these, but connected with them by occasional anastomoses, a set of branches, represented by two roots on the right side of the figure, by one large root on the other, passes to the suprarenal gland. The two on the right have apparently just lost an anastomotic connection at ' $x$,' while on the left side the plexus has been reduced to two large longitudinal vessels, each sending sprouts to the gland. This left side of the figure resembles the reconstructions of rabbit embryos made by Lewis, the longitudinal vessel representing his 'renal artery.' At this level there are no mesonephric arteries passing dorsal to the suprarenal gland. From the bottom of the longitudinal trunk on the one side, from the lower end of the lower suprarenal artery on the other, single vessels pass to the 
kidney, which lies immediately below the level of the reconstruction. From the upper end of the longitudinal vessel, on the left of the figure, a twig passes upward, possibly the future inferior phrenic artery. On the right side of the figure a similar vessel comes from a mesonephric artery, which also has two small suprarenal branches, thus showing an unusual condition. This same mesonephric artery is connected not only with a suprarenal trunk but also with the coeliac axis. Smaller aortic branches are shown, one ventral between the coeliac axis and the superior mesenteric artery, others lateral.

The permanent renal artery would have included the main trunk from aorta to kidney, arising normally in this case at the level of the second lumbar arteries, and would have had suprarenal branches. A spermatic branch would have been impossible in this embryo on the right side of the figure, highiy improbable on the other, though a roundabout connection between kidney and mesonephric artery does exist. One can readily see, however, that if more of the plexus had been retained, spermatic branches would have been easily possible. The origin of the renal artery from the coeliac axis can also be imagined in this case, and similar anastomoses between lateral or mesonephric vessels and the superior mesenteric artery would account for a renal branch of the latter vessel. Dorsal connections of the suprarenal arteries with the body-wall or with true dorsal segmental arteries can be conjectured as a persistence or extension of the plexus. The permanency of all such connections, however, must depend on the occlusion of more favorable channels.

\section{SUMMARY}

1. The anomalies of the renal artery depend on vessels present in the embryo before the aorta and its larger branches develop mesodermal coats (10-15 mm. embryos). There are no 'late branches.'

2. Certain anomalies are due to the persistence of the early renal blood supply most frequently seen with pelvic kidneys; i.e., the renal artery as a branch of the iliac, inferior mesenteric or middle sacral arteries, or from the aorta below the inferior 
mesenteric artery. Also a spermatic branch of the renal artery may be due to an early connection of a mesonephric artery with the kidney, normally lost. The kidney is probable never without arterial blood.

3. A periaortic plexus with many roots from the aorta exists in part in the mammals studied, affording an opportunity for the change of position of main aortic branches, and supplying smaller branches for possible future use.

4. The renal artery is derived from this plexus or from such parts of it as exist just before the kidney receives its capsule.

5 . The channel for the renal artery is selected mechanically, and differs in different animals with the size of the Wolffian body, the greater or lesser curvature of the rump region, and probably other physical peculiarities.

6. Channels not so convenient may be utilized if the usual channel is occluded; hence the renal variations and anomalies.

\section{BIBLIOGRAPHY}

(1) JeIdell, H. 1911 Anat. Rec., vol. 5, pp. 47-54.

(2) Evans, H. M. 1912 In Keibel and Mall's Human Embryology, vol. 2, p. 570 , et seq.

(3) Harver, R. W. 1914 Anat. Rec., vol. 8, no. 6.

(4) Broman, I. 1906 Ergebnisse der Anat. u. Entwickelung., Bd. 16, pp. 639-745; and Anat. Anz., Bd. 31, p. 94.

(5) Hochstetter, F. 1906 Hertwig's Handbuch., vol. 7, p. 116.

(6) HrLl, E. C. 1905-1906 Johns Hopkins Hosp. Bull., vol. 16, vo. 167; and vol. 17 , no. 181 .

(7) Kolster, R. 1901 Zeitschr. Morph. Anthrop., Bd. 4, pp. 179-197.

(8) Wilison, L. B. 1912 Coll. Papers St. Mary's Hosp., p. 303.

(0) Crark, E. R. 1912 Am. Jour. Anat., vol. 13, pp. 351-379, and elsewhere.

(10) Bremer, J. I. 1912 Am. Jour. Anat., vol. 13, no. 2.

(11) Dorre, A. 1889-91 Mitt. a. d. Zoöl. Stat. z. Neapel, Bd. 9.

(12) Reighard and Jennings 1901 Anatomy of the cat.

(13) Lewis, F. T. 1902 Am. Jour. Anat., vol. 1, pp. 229-244.

(14) Gerhardt, U. 1909 Das Kaninchen.

(15) Pohrman, A. G. 1905 Johns Hopkins Hosp. Bull., vol. 16, no. 176, and Amer. Medicine, vol. 7, no. 25.

(16) Lewis and PAPEZ 1914 Abstracts of Proceedings of Amer. Assoc. Anat.

(17) Goormaghtigh, U. 1914 Bull. Soc. Med. Gand., vol. 5.

(18) Canvon, W. B. 1915 Bodily changes in fear, etc. 